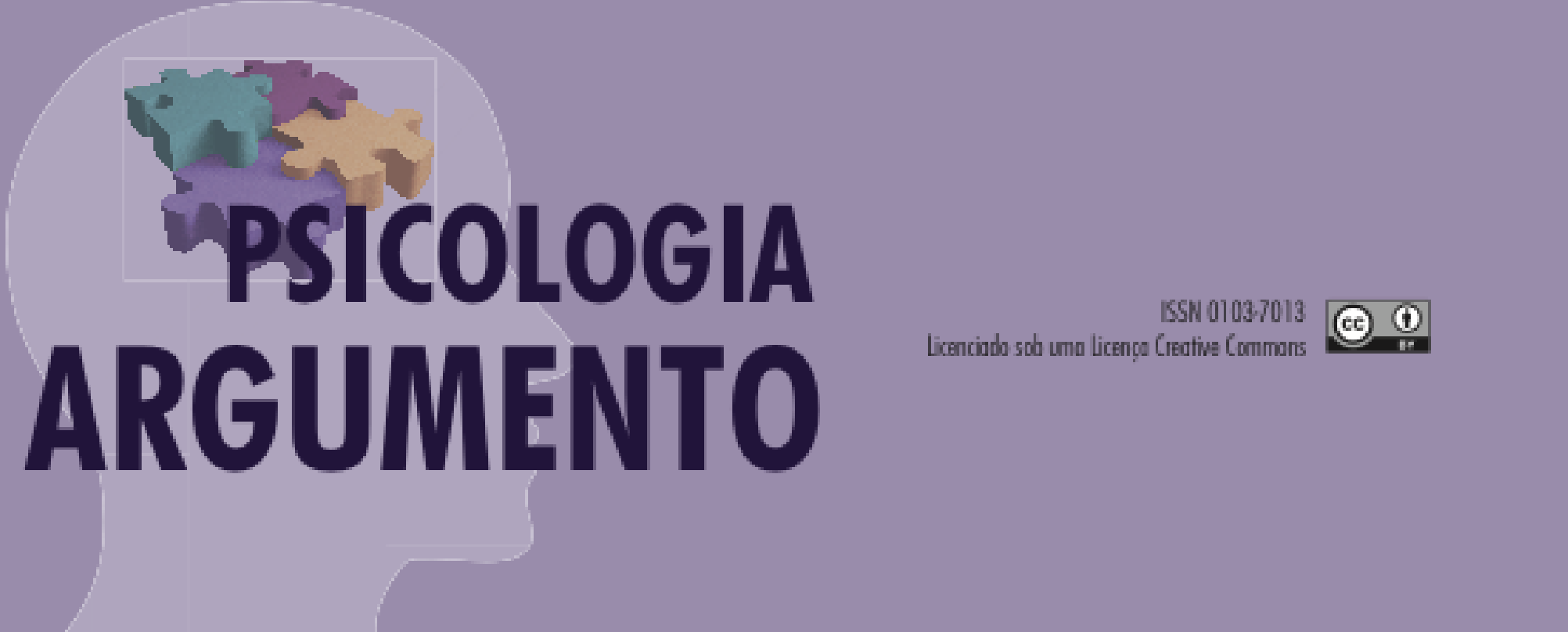

doi: http://dx.doi.org/10.7213/psicolargum.37.97.A003

\title{
Maturidade profissional como diagnóstico de necessidades de educação para carreira
}

\author{
Professional maturity as a diagnosis of career education needs
}

La madurez profesional como diagnóstico de las necesidades de educación para la carrera

Nerielen Martins Neto Fracalozzi - psicóloga, Mestre em Psicologia pelo Programa de Pósgraduação em Psicologia da Faculdade de Filosofia, Ciências e Letras de Ribeirão Preto da USP. E-mail: nerielen@yahoo.com.br ORCID: http://orcid.org/0000-0002-4989-8486

Mara de Souza Leal - psicóloga, Doutora em Psicologia pelo Programa de Pós-graduação em Psicologia da Faculdade de Filosofia, Ciências e Letras de Ribeirão Preto da USP. E-mail: marasleal@usp.br ORCID: http://orcid.org/0000-0002-9907-8814

Lucy Leal Melo-Silva - psicóloga, Professora do Departamento de Psicologia e do Programa de Pós-graduação em Psicologia da Faculdade de Filosofia, Ciências e Letras de Ribeirão Preto da USP. E-mail: lucileal@ffclrp.usp.br ORCID: https://orcid.org/0000-0002-5890-9896

\section{Resumo}

Os adolescentes, como tarefa de desenvolvimento, necessitam tomar decisões relativas aos estudos e ao trabalho, o que requer informações e maturidade. Assim, o objetivo deste estudo foi o de descrever a maturidade profissional em um grupo de adolescentes, e a partir desse diagnóstico refletir sobre as necessidades de Educação para a Carreira. Participaram do estudo 220 alunos do último ano do ensino médio (regular e técnico), com idade entre 16 e 20 anos. Os instrumentos utilizados foram: o Questionário de identificação, para registro as variáveis sociodemográficas; o Questionário de Educação para a Carreira, para identificação das necessidades de educação a carreira e o diagnóstico da maturidade; o Teste de Fotos de Profissões 
(BBT-Br), para o levantamento da produtividade nas escolhas de atividades ocupacionais. Os dados foram coletados em sala de aula, em duas escolas públicas de ensino médio. A comparação dos dados foi feita por meio do teste $t$ para amostra independentes. Os resultados não apontaram diferenças na maturidade em função das variáveis investigadas. Os alunos com maior maturidade apresentaram interesses mais amplos e diversificados, um bom sinal para a adaptação ao mundo do trabalho volátil. Porém, o conjunto da amostra apresentou baixa exploração das fontes de informações e de atividades que poderiam propiciar um maior contato com a realidade, sinalizando pistas para intervenção com base em conteúdos expressos nos itens pontuados abaixo da média, que requerem estratégias de Educação para a Carreira, com vistas à ativação de conhecimentos, habilidades e atitudes na educação básica.

Palavras-chave: educação para a carreira, escolha de carreira profissional, ensino médio, adolescentes

\begin{abstract}
Adolescents, as a developmental task, need to make decisions regarding their studies and work, which requires information and maturity. Thus, the objective of this study was to describe the professional maturity in a group of adolescents, and from this diagnosis reflect on the needs of Career Education. The study included 220 students from the last year of high school (regular and technical), aged between 16 and 20 years. The instruments used were: the identification questionnaire, to record the sociodemographic variables; the Career Education Questionnaire to identify career education needs and the diagnosis of maturity; The Profession Photo Test (BBT$B r)$, for surveying productivity in occupational activity choices. Data were collected in the classroom at two public high schools. Data were compared by independent t-test. The results showed no differences in maturity as a function of the investigated variables. The more mature students had broader and more diverse interests, a good sign for adapting to the volatile world of work. However, the sample presented low exploitation of sources of information and activities that could provide a greater contact with reality, signaling clues for intervention based on content expressed in items below the average, which require Career Education strategies, aiming at the activation of knowledge, skills and attitudes in basic education.
\end{abstract}

Keyword: career education, career choice, high school, adolescents

\begin{abstract}
Resumen
Como una tarea de desarrollo, los adolescentes necesitan tomar decisiones con respecto a sus estudios y trabajo, lo que requiere información y madurez. Por lo tanto, el objetivo de este estudio fue describir la madurez profesional en un grupo de adolescentes y, a partir de este diagnóstico, reflexionar sobre las necesidades de la educación profesional. El estudio incluyó a 220 estudiantes del último año de la escuela secundaria (regular y técnica), con edades comprendidas entre 16 y 20 años. Los instrumentos utilizados fueron: el cuestionario de identificación, para registrar las variables sociodemográficas; el Cuestionario de educación profesional para identificar las necesidades de educación profesional y el diagnóstico de madurez; y El Test de fotografia profesional (BBT-Br), para evaluar la productividad en las elecciones de actividades ocupacionales. Los datos fueron recolectados en el aula de dos escuelas secundarias públicas. Los datos se compararon mediante una prueba t independiente. Los resultados no mostraron diferencias en la madurez en función de las variables investigadas. Los estudiantes más maduros tenian intereses más amplios y diversos, una buena señal para adaptarse al volátil mundo del trabajo. Sin embargo, la muestra en su conjunto mostró una baja explotación de las fuentes de información y actividades que podrían proporcionar un mayor contacto con la realidad, señalando pistas para la intervención basada en el contenido expresado en items por debajo del promedio, que requieren estrategias de Educación para la carrera, con el objetivo de activar los conocimientos, habilidades y actitudes en la educación básica.
\end{abstract}

Palabra clave: educación profesional, elección de carrera, escuela secundaria, adolescentes 


\section{Introdução}

Para a tomada de decisão sobre estudos e trabalho as pessoas têm demandado serviços de orientação desde o início do século XX em decorrência das mudanças no trabalho derivadas do processo acelerado de industrialização. Na maior parte dos modelos de intervenção desenvolvidos no século XX, as estratégias eram mais pontuais, focalizadas na escolha, em determinados momentos da vida, conforme paradigmas da época. No final do século XX novas demandas surgiram para intervenções com vistas a auxiliar o indivíduo na execução das tarefas de desenvolvimento da carreira ao longo da vida (Watts \& Sultana, 2004). Destaca-se, neste estudo, a influência preponderante da teoria desenvolvimentista de Donald Super (1990), organizada em três segmentos: desenvolvimento, autoconceito e contextual (Lassance, Paradiso \& Silva, 2011). Por meio da investigação sobre o padrão de carreira de 100 jovens, que Super (1985) acompanhou por 20 anos, dados foram gerados possibilitando o delineamento dos principais componentes da teoria de carreira: saliência, maturação, estágios, padrões e temas de vida.

Nessa perspectiva desenvolvimentista, este estudo foi delineado com o objetivo de descrever a Maturidade vocacional, profissional ou de carreira $\left({ }^{i}\right)$ de um grupo de adolescentes, estudantes do ensino médio público (regular e técnico) e a partir desse diagnóstico apontar as necessidades de Educação para a Carreira. A Maturidade profissional, construto central desta investigação, foi definida por Super (1990) como a capacidade de um indivíduo estar apto a tomar decisões de carreira, cumprindo tarefas vocacionais pertinentes ao seu desenvolvimento biológico e social. Na década de 1980, Super (1983) propôs um modelo para a avaliação da maturidade de carreira que abarca cinco dimensões: planificação, exploração, informação, tomada de decisão e orientação para a realidade. Esse conceito é considerado o mais significativo da obra de Super em número de pesquisas (Lassance et al., 2011), com muitos estudos buscando associar a maturidade de carreira à diversas variáveis sociodemográficas e de carreira, como destacam Patton e Lokan (2001).

No que se refere aos estudos que envolvem variáveis sociodemográficas, a literatura mostra o efeito do gênero e do status socioeconômico na maturidade de carreira 
(Bae, 2017). No entanto, para Patton e Lokan (2001), essa afirmação não é consensual, a medida em que se verificam alguns estudos apontando níveis de maturidade mais altos entre alunos do sexo feminino (Bae, 2017; Ulas \& Yildirim, 2015; Zahra \& Malik, 2018) e outros não evidenciam diferenças entre os sexos (Babarovic \& Sverko, 2016; Heo \& kim, 2016; Mohammad, 2016; Karacan-Ozdemir, 2019, Talib, Salleh, Amat, Ghavifekr, \& Ariff, 2015). Com relação, especificamente, aos estudos brasileiros foram encontradas diferenças significativas na maturidade profissional em função dos sexo nas investigações de Balbinotti e Tétreau, 2006b, Aguillera (2013), Cericatto, Alves e Patias (2017) e Junqueira e Melo-Silva (2014). Quanto às condições socioeconômicas, apesar de Patton e Lokan (2001) salientarem que as investigações têm encontrado pequena ou nenhuma correlação com a maturidade de carreira como verificado no contexto brasileiro (Cattani, Teixeira, \& Ourique, 2016). Kim (2014), no contexto americano pontua que a renda familiar pode estar associada à disponibilidade de oportunidades de aprendizagem e experiências relacionadas à carreira.

Dentre outras variáveis de carreira investigadas na relação com o construto maturidade, destacam-se os interesses, construto mais investigado como apontam Leal, Fracalozzi e Melo-Silva (2010). Holland (1968) postulou que os interesses se tornariam mais diferenciados e consistentes à medida que a maturidade da carreira aumentasse, assim sendo, indivíduos com interesses mais claros apresentariam menos dificuldades em relação à tomada de decisão de carreira. No entanto, a literatura internacional aponta fracas relações entre a maturidade de carreira e a diferenciação e consistência dos interesses (Černja, Sverko, \& Babarovic, 2018). Para Achtnich (1991), os interesses profissionais, ou inclinações, traduzem a preferência por determinadas atividades, ambientes, objetos e instrumentos de trabalho. Em um estudo sobre a associação entre maturidade para a escolha profissional, avaliada por meio da Escala de Maturidade para a Escolha Profissional (EMEP) (Neiva, 1999) e os interesses, mensurados por meio do Teste de Fotos de Profissões (BBT-Br) (Achtnich, 1991), Noce (2008) destaca que o nível de maturidade mais elevado se relaciona com o número maior de escolhas de fotos positivas no BBT-Bt (produtividade), sinalizando maior abertura para as possibilidades profissionais. 
Investigações sobre a relação entre a maturidade e outras dimensões psicológicas são relevantes para a compreensão dos problemas no domínio da Orientação Profissional e de Carreira. Mensurar os níveis de maturidade de jovens em situação de tomada de decisão de carreira tem sido um dos objetivos de pesquisadores, visando a compreensão de processos decisórios. E, também, dos profissionais da orientação, visando a definição de estratégias de intervenção que atendam às demandas específicas dos usuários de serviços, como apontam Lassance et al. (2007) e Antunes et al. (2009). Um dos objetivos das intervenções do conselheiro de carreira é antecipar as tarefas evolutivas (Super, 1990) e, assim, poder auxiliar os adolescentes na formação de atitudes e competências para as decisões sobre a continuidade dos estudos e a preparação para o trabalho futuro, assim como, desenvolver a confiança para lidar com tarefas de desenvolvimento de carreira (Leal, Melo-Silva, \& Teixeira, 2015).

Uma das modalidades de intervenção é a Educação para a Carreira, também objeto deste artigo. Com base nas premissas da Teoria Desenvolvimentista de Carreira (Super, 1990), de que a carreira é construída ao longo da vida, e nas necessidades de conectar educação e trabalho (Herr, 2001) surgiu, nos EUA, o modelo de Educação para a Carreira. Essa modalidade de intervenção sistemática de Orientação para a Carreira realizada em contexto escolar, tem por finalidade propiciar situações que estimulam o desenvolvimento de atitudes de autogestão e de habilidades de planejamento e de tomada de decisão de carreira (Savickas, 2004). De acordo com Marland (1972), a Educação para a Carreira compreende um conjunto de experiências educativas, na perspectiva do desenvolvimento integral do aluno, de modo a que ele alcance a independência financeira, o bem-estar pessoal e o senso de responsabilidade em relação ao trabalho. Para Hoyt (2005), a Educação para a Carreira pode ser entendida como um esforço da comunidade e do sistema de ensino em promover a aquisição de competências necessárias para o desenvolvimento de carreira e, também, atribuir sentido significativo do trabalho na vida das pessoas, independentemente de remuneração. Assim, Munhoz, Melo-Silva e Audibert (2016), no contexto brasileiro, salientam a importância de se promover intervenções no contexto educativo, de forma, a integrar conteúdos de carreira no currículo escolar desde os anos iniciais, visando a promoção de competências de autogestão e a inserção efetiva do indivíduo no mundo do trabalho. A oferta de programas voltados para o 
desenvolvimento de carreira na educação básica requer investimento em políticas públicas como apontam Melo-Silva, Munhoz e Leal (2019).

Considerando o anteriormente exposto, neste estudo, indaga-se quais os níveis de maturidade profissional em uma amostra de estudantes brasileiros. Haveria necessidade de se pensar em estratégias de Educação para a Carreira? Assim, este estudo objetiva descrever a maturidade profissional de uma amostra de jovens provenientes do ensino médio, em função das variáveis sexo, nível socioeconômico, procedência escolar e interesses profissionais e levantar indicadores sobre a necessidade de Educação para a Carreira.

\section{Método}

\section{Participantes}

A amostra, de conveniência, é composta por alunos do último ano do ensino médio público, do período diurno, com idade entre 16 e 20 anos. Totalizam 220 participantes, de ambos os sexos, de duas escolas de nível médio (uma regular e outra técnica) de uma cidade do interior do Estado de São Paulo. No caso dos estudantes do ensino técnico, eles se encontravam matriculados em diferentes cursos, a saber, Administração, Design de Interiores, Edificações, Eletrônica, Eletrotécnica, Mecatrônica, Nutrição e Dietética, e Secretariado. Cerca de metade dos estudantes, tanto ensino médio regular quanto do técnico afirmavam estar trabalhando e/ou já terem trabalhado.

A amostra do ensino médio regular é constituída por 131 alunos, sendo 91 do sexo feminino e 40 do sexo masculino. A amostra do ensino técnico é constituída por 89 participantes, sendo 56 do sexo feminino e 33 do sexo masculino. De acordo com o Critério de Classificação Econômica Brasil (ABEP, 2012), a maioria dos alunos, em ambas as escolas, pertenciam ao nível socioeconômico B.

\section{Instrumentos}

1. Questionário de identificação, construído pelo grupo de pesquisa com o objetivo de reunir informações sociodemográficas, de trabalho e escolha da carreira, incluindo o Critério de Classificação Econômica Brasil (CCEB), instrumento da 
Associação Brasileira de Empresas de Pesquisa (ABEP) que objetiva estimar o poder de compra das pessoas e famílias urbanas (ABEP, 2012).

2. Questionário de Educação para a Carreira (QEC), destinado à avaliação da maturidade vocacional de alunos do ensino médio e ao levantamento de necessidades de Educação para a Carreira. O QEC, instrumento principal deste estudo, é formado por 96 itens divididos em duas escalas afetivas, a primeira, unidimensional, denominada "Sentido e Importância do trabalho" (com 22 itens) e a segunda, "Preparação à Carreira", subdividida em seis subescalas, a saber, "Passos Efetuados" (13 itens), "Fatores Considerados" (17 itens), "Profissão Preferida" (9 itens), "Pesquisa e Conservação de Emprego" (11 itens), "Pessoas e Fontes Consultadas" (11 itens) e "Atividades Realizadas" (13 itens). Cabe ressaltar que as quatro primeiras subescalas referem-se às tarefas de planificação da carreira enquanto que as duas últimas relacionam-se às tarefas de exploração. Obtém-se, dessa forma, um escore específico para cada uma das sete dimensões e um escore global. Trata-se de uma escala do tipo Likert graduada em quatro pontos. As respostas vão de "completamente em desacordo" a "completamente de acordo", ou "nunca" a "frequentemente", ou "conheço pouquíssimo" a "conheço muitíssimo", ou ainda de "pouquíssimo" a "muitíssimo", dependendo da subescala a que se referem. A versão utilizada neste estudo é a de Aguillera (2013) adaptado de Balbinotti e Tétreau (2006a) para uso no Brasil. O instrumento apresenta propriedades psicométricas satisfatórias com o Alpha de Crombach variando entre 0,77 a 0,89 na amostra investigada.

3. Teste de Fotos de Profissões ou Berufsbilder-Test (BBT). Consiste em um método projetivo de avaliação dos interesses, composto por 96 fotos de profissionais retratados em situação de trabalho. $\mathrm{O}$ instrumento investiga a estrutura de inclinação motivacional do indivíduo por meio da identificação deste com a atividade do trabalho, revelando informações sobre o seu perfil de interesses, que é obtido por meio da classificação das fotos em (a) escolhas positivas (fotos que agradam), (b) escolhas negativas (fotos que não agradam), e (c) escolhas neutras (fotos que suscitam indiferença no sujeito). Utilizado neste estudo com o objetivo de verificar se o número de escolhas positivas (produtividade) apresenta alguma associação com o nível de maturidade. 
Procedimentos para a coleta e análise dos dados

Este estudo faz de um projeto maior que investigou educação para a carreira, interesses profissionais e autoeficácia em estudantes do ensino médio público, regular e técnico, por meio de dois subprojetos (Leal, 2013; Fracalozzi, 2014). Este estudo utilizou a base de dados da pesquisa de mestrado de Fracalozzi (2014). Após aprovação do projeto pelo Comitê de Ética (Processo No 572/2011-2011.1.1169.59.1.) e dos responsáveis pelas escolas, foi realizado o contato com os alunos em sala de aula para explicar a pesquisa e, em caso de aceite, para que formalizassem sua participação voluntária por meio do Termo de Assentimento Livre e Esclarecido (TALE), que foi afirmado pelos pais, por meio da assinatura do Termo de Consentimento Livre e Esclarecido (TCLE), nos casos de alunos com idade inferior a 18 anos. Foram entregues aos alunos o TCLE e a Ficha de identificação e o Critério de Classificação Econômica Brasil (ABEP, 2012). Em seguida, foram agendados os horários para a coleta em sala de aula, de acordo com a disponibilidade das escolas. A coleta de dados foi realizada por duas psicólogas treinadas para a aplicação dos instrumentos. Devido ao seu caráter projetivo, o BBT-Br foi o primeiro instrumento a ser aplicado. As fotos foram apresentadas aos alunos em slides com tempo controlado de 15 segundos para cada foto, depois foi realizada a aplicação do QEC, com duração aproximada de 30 minutos. Salienta-se que os dois instrumentos foram aplicados conforme instruções dos referidos instrumentos.

As informações foram inseridas nas bases de dados, uma com os dados de identificação e os resultados do QEC e outra plataforma específica para o BBT-Br. As análises descritivas dos dados envolveram o cálculo das médias e desvio padrão para cada uma das sete escalas e subescalas do questionário e também para a pontuação total, com base na somatória dos escores dos participantes. A fim de verificar possíveis diferenças estatísticas entre os resultados referentes à maturidade profissional, os dados foram submetidos ao teste $\mathrm{t}$ de Student para amostras independentes em função do sexo, procedência escolar e nível socioeconômico para as médias totais dos escores de cada uma das subescalas e para a escala total, adotando-se um nível de significância $\mathrm{p} \leq 0,05$. Vale pontuar que, para a análise da maturidade em função do nível socioeconômico, os estratos socioeconômicos foram agrupados. O grupo denominado $\mathrm{AB}$ foi formado por adolescentes cuja renda familiar foi classificada nos níveis A1, A2, B1 ou B2 e o grupo 
$\mathrm{CD}$ foi formado pelos jovens classificados nos grupos $\mathrm{C} 1, \mathrm{C} 2$ ou $\mathrm{D}$, de acordo com o resultado do Critério de Classificação Econômica Brasil (ABEP, 2012).

Foram analisadas também as médias das pontuações da amostra total em cada um dos 96 itens do QEC para avaliar em quais escalas e subescalas é possível identificar as principais necessidades de Educação para a Carreira dos estudantes. Para isso, as médias de pontuação de cada item foram comparadas à média geral de pontuação total do instrumento em toda a amostra $(2,92)$, fornecendo assim pistas para a intervenção.

Para verificar se existem diferenças no nível de maturidade de carreira em função dos índices de produtividade do BBT-Br, escolhas de interesses profissionais realizadas como positivas, negativas e neutras, os participantes foram divididos em dois grupos, um com escore mais baixo no QEC (percentil 25) e outro com maiores pontuações (percentil 75). A comparação foi realizada por meio do teste $t$ de student, considerando um nível de significância igual ou menor a 0,05 .

\section{Resultados}

Foram realizados cálculos estatísticos visando identificar possíveis diferenças entre os alunos em função das variáveis sexo, nível socioeconômico e procedência escolar. Não foram verificadas diferenças estatisticamente significadas nas subescalas e na escala total da maturidade de carreira, conforme mostra os resultados em função das variáveis sexo (meninas: $M=280,54, D P=31,28$; meninos: $M=281,47, D P=33,18$; $t(218)=0,123, p=0,902)$, nível socioeconômico (AB: $M=291,15, D P=32,66$; CD: $M$ $=280,12, D P=30,07 ; t(218)=0,217, p=0,828)$ e procedência escolar (ensino médio regular: $M=281,97 ; D P=30,80$; ensino técnico: $M=279,19, D P=33,45 ; t(218)=$ $0,634, p=0,527)$. Visando identificar as principais necessidades de educação para a carreira dos estudantes, buscou-se verificar quais itens obtiveram média inferior à média geral no teste (2,92). Esses itens de pontuação abaixo da média, que são relevantes neste estudo com vistas a buscar pistas para a intervenção, são apresentados na Tabela 1.

A partir dos resultados da Tabela 1, pode-se depreender que as maiores pontuações dos participantes no QEC estão relacionadas ao Sentido e Importância do Trabalho, dimensão na qual somente dois itens pontuaram abaixo da média geral da escala (9\%). 
Tabela 1

Itens abaixo da média na subescala Sentido e Importância do Trabalho

\begin{tabular}{|c|c|c|c|c|c|c|}
\hline Subescala & Itens & Enunciado & $\mathrm{M}$ & DP & $\begin{array}{c}\% \text { itens } \\
>\text { média por } \\
\text { subescala }\end{array}$ & $\begin{array}{c}\% \text { itens } \\
>\text { média por } \\
\text { tarefa }\end{array}$ \\
\hline \multirow{2}{*}{$\begin{array}{l}\text { Sentido e } \\
\text { Importância } \\
\text { do Trabalho }\end{array}$} & 8 & $\begin{array}{l}\text { Eu sempre posso fazer uma ou outra coisa que eu } \\
\text { goste não importando o trabalho que eu faça }\end{array}$ & 2,77 & $\begin{array}{c}0,79 \\
5\end{array}$ & \multirow[b]{2}{*}{9,0} & \multirow[b]{2}{*}{9,0} \\
\hline & 12 & $\begin{array}{c}\text { O trabalho é necessário para resolver os } \\
\text { problemas de nossa sociedade }\end{array}$ & 2,87 & $\begin{array}{c}0,82 \\
1\end{array}$ & & \\
\hline
\end{tabular}

A Tabela 2, a seguir, apresenta os itens abaixo da média nas subescalas

relacionadas às tarefas de planificação da carreira.

Tabela 2

Itens abaixo da média nas subescalas de planificação da carreira

\begin{tabular}{|c|c|c|c|c|c|c|}
\hline Subescala & Itens & Enunciado & M & DP. & $\begin{array}{c}\% \text { itens } \\
>\text { média geral } \\
\text { por subescala }\end{array}$ & $\begin{array}{l}\% \text { itens } \\
>\text { média geral } \\
\text { por tarefa }\end{array}$ \\
\hline $\begin{array}{l}\text { Passos } \\
\text { Efetuados }\end{array}$ & 29 & $\begin{array}{l}\text { Participar de diferentes atividades para melhor } \\
\text { me conhecer }\end{array}$ & 2,91 & 0,849 & 7,6 & 42,0 \\
\hline \multirow{9}{*}{$\begin{array}{c}\text { Aspectos } \\
\text { Considerados }\end{array}$} & 42 & $\begin{array}{l}\text { A formação necessária para realizar meus } \\
\text { projetos de orientação }\end{array}$ & 2,82 & 0,840 & \multirow{9}{*}{53,0} & \\
\hline & 43 & $\begin{array}{l}\text { Um bom número de possibilidades de formação } \\
\text { profissional depois do segundo grau }\end{array}$ & 2,86 & 0,789 & & \\
\hline & 44 & As leis e os regulamentos do trabalho & 2,35 & 0,845 & & \\
\hline & 45 & $\begin{array}{l}\text { O funcionamento da economia em relação ao } \\
\text { mercado de trabalho }\end{array}$ & 2,41 & 0,853 & & \\
\hline & 46 & $\begin{array}{c}\text { A influência das mudanças tecnológicas sobre } \\
\text { minha carreira futura }\end{array}$ & 2,73 & 0,856 & & \\
\hline & 47 & $\begin{array}{l}\text { As tendências atuais e futuras do emprego nos } \\
\text { diversos setores do mercado de trabalho }\end{array}$ & 2,62 & 0,804 & & \\
\hline & 48 & $\begin{array}{c}\text { Os meios de enfrentar uma situação de } \\
\text { desemprego no curso de uma vida de trabalho }\end{array}$ & 2,47 & 0,835 & & \\
\hline & 49 & $\begin{array}{c}\text { A experiência necessária para diversos } \\
\text { empregos }\end{array}$ & 2,38 & 0,917 & & \\
\hline & 52 & $\begin{array}{l}\text { As exigências de admissão nos programas ou } \\
\text { escolas onde eu poderei ir no próximo ano }\end{array}$ & 2,63 & 0,883 & & \\
\hline \multirow{3}{*}{$\begin{array}{l}\text { Profissão } \\
\text { Preferida }\end{array}$} & 53 & As tarefas exercidas & 2,87 & 0,743 & \multirow[t]{3}{*}{33,3} & \\
\hline & 59 & As condições de trabalho & 2,86 & 0,849 & & \\
\hline & 60 & O salário de base oferecido & 2,63 & 0,903 & & \\
\hline \multirow{8}{*}{$\begin{array}{c}\text { Pesquisa } \\
\text { Conservação } \\
\text { Emprego }\end{array}$} & 62 & Onde procurar um emprego & 2,64 & 0,811 & \multirow{8}{*}{72,7} & \\
\hline & 63 & $\begin{array}{l}\text { Como preencher um formulário de pedido de } \\
\text { emprego }\end{array}$ & 2,46 & 0,829 & & \\
\hline & 64 & $\begin{array}{c}\text { Como escrever uma carta de pedido de } \\
\text { emprego }\end{array}$ & 2,25 & 0,859 & & \\
\hline & 65 & Como preparar meu currículo & 2,79 & 0,838 & & \\
\hline & 66 & $\begin{array}{c}\text { Como me preparar para uma entrevista de } \\
\text { emprego }\end{array}$ & 2,85 & 0,811 & & \\
\hline & 68 & $\begin{array}{l}\text { Como os empregadores fazem para contratar } \\
\text { alguém }\end{array}$ & 2,47 & 0,867 & & \\
\hline & 69 & $\begin{array}{l}\text { O que os empregadores esperam do novo } \\
\text { funcionário }\end{array}$ & 2,85 & 0,822 & & \\
\hline & 70 & $\begin{array}{l}\text { Quais são as qualidades pessoais que os } \\
\text { empregadores consideram as mais importantes } \\
\text { para contratar alguém }\end{array}$ & 2,72 & 0,817 & & \\
\hline
\end{tabular}


Verifica-se que $42 \%$ dos itens das subescalas relacionadas às tarefas de planificação da carreira apresentam-se abaixo da média geral obtida por meio do QEC com a amostra. Ao analisar as subescalas separadamente, verifica-se menor porcentagem de itens abaixo da média na subdimensão Passos Efetuados. As subescalas com uma maior porcentagem de itens abaixo da média geral são a Pesquisa e Conservação de Emprego, na qual 8 dos 11 itens apresentaram média inferior à média geral do teste (72,7\%) e os Aspectos Considerados, na qual mais da metade dos itens também se apresentam abaixo da média (53\%). Na Tabela 3, a seguir, são apresentados os itens abaixo da média nas subescalas relacionadas às tarefas de exploração da carreira.

Tabela 3

Itens abaixo da média nas subescalas de exploração da carreira

\begin{tabular}{|c|c|c|c|c|c|c|}
\hline Subescala & $\begin{array}{l}\text { Ite } \\
\text { ns }\end{array}$ & Enunciado & $\mathrm{M}$ & DP. & $\begin{array}{c}\% \text { itens } \\
>\text { média } \\
\text { geral por } \\
\text { subescala }\end{array}$ & $\begin{array}{c}\% \text { itens } \\
>\text { média } \\
\text { geral por } \\
\text { tarefa }\end{array}$ \\
\hline \multirow{9}{*}{$\begin{array}{l}\text { Pessoas e } \\
\text { fontes } \\
\text { consultadas }\end{array}$} & \multicolumn{2}{|r|}{ De meus amigos } & 2,70 & ,849 & \multirow[t]{9}{*}{81,8} & \multirow[t]{9}{*}{91,6} \\
\hline & 75 & De outros membros de minha família & 2,53 & 1,039 & & \\
\hline & 76 & $\begin{array}{l}\text { De um conselheiro de orientação } \\
\text { profissional }\end{array}$ & 2,07 & 1,103 & & \\
\hline & 77 & De um professor de educação à carreira & 2,32 & 1,126 & & \\
\hline & 78 & De outros professores & 2,30 & 1,021 & & \\
\hline & 79 & De responsáveis de outras escolas & 1,74 & ,926 & & \\
\hline & 80 & De livros, brochuras ou jornais & 2,68 & ,930 & & \\
\hline & 81 & $\begin{array}{l}\text { De vídeos, de filmes ou programas de } \\
\text { televisão }\end{array}$ & 2,70 & ,983 & & \\
\hline & 83 & $\begin{array}{l}\text { De programas escolares (ou universitários) } \\
\text { ou guias de profissões (revistas) }\end{array}$ & 2,67 & ,988 & & \\
\hline \multirow[t]{13}{*}{$\begin{array}{l}\text { Atividades } \\
\text { Realizadas }\end{array}$} & 84 & $\begin{array}{l}\text { De conversas com trabalhadores exercendo } \\
\text { diferentes empregos }\end{array}$ & 2,31 & ,981 & \multirow[t]{13}{*}{100,0} & \\
\hline & 85 & De encontros com empregadores & 1,89 & ,923 & & \\
\hline & 86 & $\begin{array}{l}\text { De conferências de representantes do } \\
\text { mundo do trabalho }\end{array}$ & 1,91 & ,934 & & \\
\hline & 87 & De meus cursos em diferentes matérias & 2,68 & ,924 & & \\
\hline & 88 & De cursos de educação à carreira & 2,31 & 1,044 & & \\
\hline & 89 & De minhas atividades paralelas à escola & 2,65 & 969 & & \\
\hline & 90 & $\begin{array}{l}\text { De atividades como "um-dia-de-trabalho" } \\
\text { ou de seções de informações profissionais }\end{array}$ & 2,19 & 1,090 & & \\
\hline & 91 & $\begin{array}{c}\text { De visitas em escolas, colégios ou } \\
\text { universidades }\end{array}$ & 2,09 & 1,009 & & \\
\hline & 92 & De minhas experiências de trabalho em casa & 2,37 & 968 & & \\
\hline & 93 & De minha experiência de trabalho fora & 2,37 & 1,108 & & \\
\hline & 94 & De estágios em lugares de trabalho & 2,02 & 1,156 & & \\
\hline & 95 & De visitas em empresas ou indústrias & 2,10 & 1,090 & & \\
\hline & 96 & $\begin{array}{c}\text { De minhas observações de pessoas no } \\
\text { trabalho }\end{array}$ & 2,85 & ,993 & & \\
\hline
\end{tabular}


O maior número de itens com pontuação abaixo da média foi encontrado nas duas últimas subescalas (91,6\%). Na subescala Pessoas e Fontes Consultadas $81,8 \%$ dos itens se apresentam abaixo da média geral no instrumento. Os únicos dois itens nessa escala que pontuaram acima da média dizem respeito à busca majoritária por informações com os pais e na internet. Por fim, na subescala Atividades Realizadas, a média de todos os itens foi inferior à média geral, o que evidência que os jovens da amostra analisada não estão obtendo ajuda e/ou informação por meio das atividades listadas. A seguir, a Tabela 4 mostra os resultados da comparação dos níveis de maturidade de carreira em função do número de escolhas de fotos de pessoas exercendo atividades ocupacionais que expressam os interesses ou inclinação profissional no BBT-Br (fotos escolhidas positivamente, negativamente ou neutras/indiferentes).

Tabela 4

Comparação do nível de maturidade em função da produtividade (escolhas positivas) no BBT.

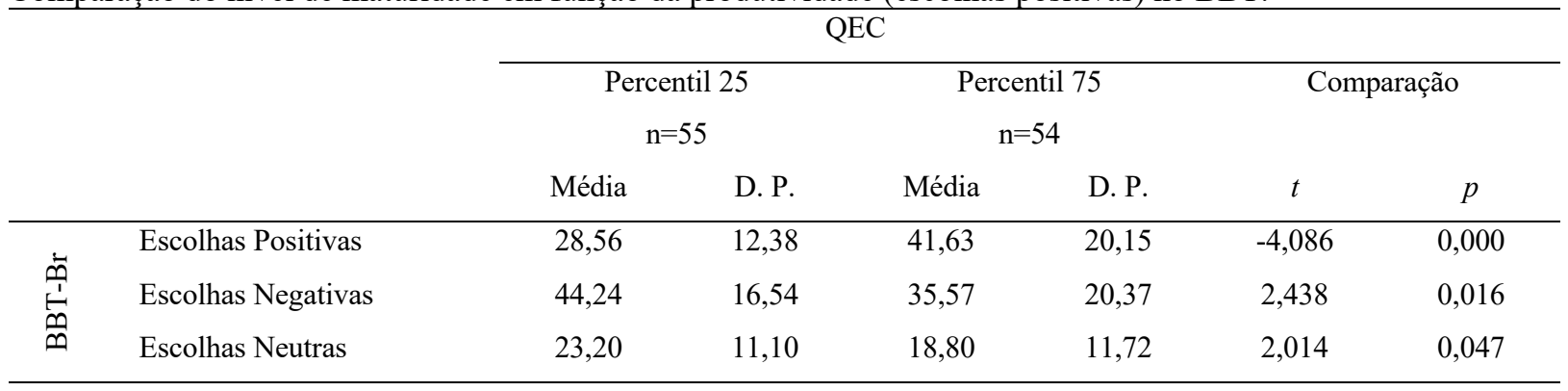

A comparação dos alunos de menor nível de maturidade de carreira dessa amostra com os de maior nível evidencia diferenças significativas nas escolhas positivas, negativas e neutras. Observa-se que os adolescentes com maior nível de maturidade vocacional realizam mais escolhas positivas e menos escolhas negativas e neutras.

\section{Discussão}

Neste estudo, a comparação da maturidade de carreira na escala total e nas subescalas do QEC não apontou diferenças estatisticamente significativas em função do sexo. Resultado que se assemelha ao encontrado em achados internacionais (Babarovic \& Sverko, 2016; Heo \& kim, 2016; Mohammad, 2016; Talib et al. 2015), no entanto, difere dos resultados encontrados por estudo nacional de Aguillera (2013) e Balbinotti e Tétreau (2006b) com o uso de QEC e de Junqueira e Melo-Silva (2014) e Cericatto et al. 
(2017) com o uso de outro instrumento, a Escala de Maturidade para a Escolha Profissional (EMEP). A ausência de diferenças estatisticamente significativas nos níveis de maturidade vocacional em função do nível socioeconômico familiar está em consonância com achados de pesquisas internacionais. Segundo Patton e Lokan (2001), a literatura internacional têm encontrado baixa ou nenhuma correlação entre maturidade vocacional e nível socioeconômico em adolescentes e em universitários.

Por sua vez, neste estudo, a ausência de diferenças estatisticamente significativas na maturidade profissional, em função do tipo de escola de ensino médio, regular ou técnico, foi uma constatação surpreendente. Era esperado encontrar um maior grau de maturidade profissional a favor dos alunos da escola de ensino técnico, já que eles já haviam passado por experiência de trabalho por meio dos estágios $(49,4 \%)$, o que supõese que lhes garantiria maior maturidade. Uma possível explicação para a ausência de diferenças, pode estar no fato da amostra proveniente do ensino médio regular possuir alunos que também já passaram por experiências de trabalho $(48,8 \%)$, sendo, o número de estudantes trabalhadores semelhantes nas duas instituições. Outra explicação pode estar nas características das escolas, ambas públicas e com alunos trabalhadores. Em comum elas são caracterizadas como referência na cidade.

Em relação as necessidades de Educação para a Carreira dos estudantes, pode-se depreender a partir dos resultados, que de uma maneira geral, os adolescentes da amostra reconhecem o sentido e a importância do trabalho e se engajam medianamente no planejamento do que pode ser feito para conhecer melhor sobre o mundo de trabalho e das profissões. Contudo, estão explorando pouco suas possibilidades em termos de fontes de informações e de atividades que poderiam propiciar um maior contato com a realidade a fim de permitir condições mais favoráveis de se impetrar uma escolha mais consciente.

Nesse sentido, as baixas pontuações em itens das subescalas de tarefas de planificação e exploração de carreira poderão constituir-se em pistas para intervenções futuras. Intervenções que abordem, por exemplo, os meios de enfrentar uma situação de desemprego, possibilidades e exigências de admissão em programas ou escolas, a maneira de se preparar para uma entrevista, as tarefas exercidas e as condições de trabalho, a influência das mudanças tecnológicas sobre a carreira, as tendências de emprego, visitas às escolas, universidades, empresas e conferências com representantes do mundo do 
trabalho, poderiam auxiliar os jovens a se prepararem melhor para enfrentar o mercado de trabalho e suas vicissitudes, facilitando seu processo de autoconhecimento e desenvolvimento de carreira. Vale salientar que, trabalhando os referidos conteúdos por meio de intervenções, a escola estará colaborando para o desenvolvimento da maturidade profissional de seus alunos, que como demonstrado neste estudo e, em investigação anterior (Noce, 2008) está relacionada a ampliação dos interesses de forma mais realista e a maior abertura para as possibilidades profissionais.

\section{Considerações finais}

Em síntese, com base nos dados, algumas das pistas para intervenções em Educação para a Carreira com adolescentes do ensino médio consistem na oferta de atividades que permitam atribuir sentido e importância ao trabalho, que possibilitem a ampliação da consciência sobre alternativas de trabalho e sobre a necessidade de resolver problemas sociais, o que possibilitaria aos estudantes do final do ensino médio maior engajamento na escola por atribuir sentido aos estudos como base de construção do futuro.

Objetivando a planificação da carreira, destaca-se como eixos temáticos da intervenção: aprofundamento no autoconhecimento; maior clareza dos caminhos para a formação e preparação profissional disponíveis no território; aprofundamento em questões do trabalho: legislação, economia e mercado de trabalho, emprego/desemprego, condições salariais; como se candidatar a emprego, preparar currículo, preencher formulários e treinar para entrevista de emprego; onde procurar trabalho; como escrever carta ou mensagem pedindo emprego; saber quais são as expectativas dos empregadores e competências (habilidades) esperadas; saber mais sobre as mudanças tecnológicas; como utilizar mais fontes de informação, como se preparar para ter experiências em estágios, efetivar visitas a empresas e observações de pessoas no trabalho. Conhecer-se e conhecer o mundo do trabalho continua a ser essencial na vida, porém, no século XXI novos desafios estão colocados no sentido de resolução de problemas complexos em cenários e contextos de falta de trabalho digno e com vertiginosas mudanças decorrentes das novas tecnologias. 
No mundo contemporâneo, espera-se das novas gerações que estejam aptas a lidar com mudanças, que sejam proativas e se reinventem frente ao mundo volátil, incerto, complexo e ambíguo. Nesse sentido, os programas de Educação para a Carreira podem ser estratégias úteis para auxiliar o adolescente no desenvolvimento de carreira, principalmente se as ações forem implementadas na escola desde a infância. Intervenções que busquem investir na preparação do jovem para enfrentar os desafios que se impõem ao longo do seu desenvolvimento de carreira, fornecendo informações e ajudando-o a desenvolver habilidades que sejam úteis em sua trajetória de vida são essenciais para prepará-lo para a vida e o mundo do trabalho, e atribuindo maior sentido aos estudos e ao trabalho na construção da vida/carreira.

\section{Agradecimentos}

Agradecemos à agência de fomento CAPES, pelo apoio recebido por meio do financiamento do Programa de bolsas da CAPES. Este estudo é parte de uma investigação maior que resultou na dissertação de mestrado da primeira autora, orientada pela terceira.

\section{Referências}

Achtnich, M. (1991). O BBT-Teste de Fotos de Profissões: método projetivo para a clarificação da inclinação profissional. Trad. José Ferreira Filho. São Paulo: CETEPP.

Antunes, J. B., Melo-Silva, L. L, Faria, L., Taveira, M. C., Repetto-Talavera, E., Soto, N. M. Et al. (2009). Competências do orientador profissional Brasileiro. Revista Española de Orientación y Psicopedagogía, v. 20, p. 99-108.

Aguillera, F. (2013). Projeto de vida e preparação para a carreira de jovens aprendizes: Da realidade à intervenção (Tese de doutorado não publicada). Universidade de São Paulo, Ribeirão Preto, SP. 
Associação Brasileira de Empresas de Pesquisa. (2012). Critério de Classificação Econômica Brasil. Recuperado

de http://www.abep.org/novo/Content.aspx?ContentID=301

Balbinotti, M. A. A., \& Tétreau, B. (2006a). Questionário de educação à carreira: Propriedades psicométricas da versão brasileira e comparação transcultural. Revista Brasileira de Orientação Profissional, 7(2), 49-63. Retirado de http://pepsic.bvsalud.org/scielo.php?script=sci_arttext\&pid=S167933902006000200006

Bae, S.-M. (2017). An analysis of career maturity among Korean youths using latent growth modeling. School Psychology International, 38(4), 434449. https://doi.org/10.1177/0143034317709527

Babarovic, T., \& Sverko, I. (2016). Vocational Development in Adolescence: Career construction, Career Decision-Making Difficulties And Career adaptability of Croatian High School Students. Phimenjena Psihologija, 9 (4), 429-448.

Balbinotti, M. A. A., \& Tétreau, B. (2006b). Níveis de maturidade vocacional de alunos de 14 a 18 anos do Rio Grande do Sul. Psicologia em Estudo, 11(3), 551-560. Retirado de $\quad$ http://www.scielo.br/scielo.php?script $=$ sci arttext\&pid=S141373722006000300011\&lng=en\&nrm=iso\&tlng=pt.

Cattani, B. C., \& Teixeira, M. A. P., \& Ourique, L. R. (2016). Maturidade de carreira e nível socioeconômico em estudantes do ensino médio. Gerais: Revista Interinstitucional de Psicologia, 9(1), 67-77. Recuperado em 17 de outubro de 2019, de http://pepsic.bvsalud.org/scielo.php?script=sci_arttext\&pid=S1983$82202016000100006 \& \operatorname{lng}=$ pt\&tlng $=$ pt.

Cericatto, C., Alves, C. F., Patias, N. D. (2017). A Maturidade para a Escolha Profissional em Adolescentes do Ensino Médio. Revista de Psicologia da IMED, 9 (1), 23-37. 
Černja I., Šverko I., \& Babarović T. (2018) Career Maturity Indicators in Adolescence: Convergence of Different Measures. In: Cohen-Scali V., Rossier J., Nota L. (Eds.), New perspectives on career counseling and guidance in Europe. Springer, Cham.

Fracalozzi, N. M. N. (2012). Educação para a carreira e interesses profissionais em estudantes do ensino médio regular e técnico (Dissertação de mestrado não publicada). Universidade de São Paulo, Ribeirão Preto, SP.

Heo, G., \& Kim, T. (2016). Autoregressive Cross-Lagged Modeling of the Reciprocal Longitudinal Relationship Between Self-Esteem and Career Maturity. Jorunal of Carrer Development, 43(3), 273-288.

Herr, E. L. (2001). Career development and its practice: A historical perspective. Career Development Quarterly, 49(3), 196-211. DOI: 10.1002/j.2161-0045.2001.tb00562.x

Holland, J. L. (1968). Explorations of a theory of vocational choice: VI. A longitudinal study using a sample of typical college students. Journal of Applied Psychology, 52(1, Pt.2), 1-37. https://doi.org/10.1037/h0025350

Hoyt, K. B. (2005). Career education as a federal legislative effort. In K. B. Hoyt (Org.), Career education: History and future (pp. 3-74). Oklahoma City, OK: National Career Development Association.

Junqueira, M. L., \& Melo-Silva, L. L. (2014). Maturidade para a escolha de carreira: estudo com adolescentes de um serviço-escola. Revista Brasileira de Orientação Profissional, 15(2), 187-199. Recuperado em 13 de outubro de 2019, de http://pepsic.bvsalud.org/scielo.php?script=sci_arttext\&pid=S167933902014000200009\&lng=pt\&tlng=pt.

Karacan-Ozdemir, N. (2019). Associations between career adaptability and career decision-making difficulties among Turkish high school students. International Journal for Educational and Vocational Guidance. 19 (3), 475-495. 
Kim, M. (2014). Family background, students' academic self-efficacy, and students' career and life success expectations. International Journal for the Advancement of Counselling,36(4), 395-407.

Lassance, M. C. P. ; Melo- Silva, Lucy Leal ; Bardagi, M. P. ; Paradiso, Â. C. . Competências do Orientador Profissional: uma proposta brasileira com vistas à formação e certificação. Revista Brasileira de Orientação Profissional, v. 8, p. 87-94, 2007.

Lassance, M. C. P., Paradiso, A. C., \& Silva, C. B. (2011). Terceira demanda-chave para a orientação profissional: Como ajudar o indivíduo a desenvolver sua carreira? Enfoque desenvolvimentista e evolutivo. In M. A. Ribeiro \& L. L. Melo-Silva (Orgs.), Compêndio de orientação profissional e de carreira: Perspectivas históricas e enfoques teóricos clássicos e modernos (Vol. 1, pp. 135-166). São Paulo, SP: Vetor.

Leal, M. S. (2013). Autoeficácia percebida em desenvolvimento de carreira e interesses profissionais em estudantes do ensino médio regular e técnico (Dissertação de mestrado não publicada). Universidade de São Paulo, Ribeirão Preto, SP.

Leal, M. S., Fracalozzi, N. M. N., Melo-Silva, L.L. (2010). Produção científica em congressos brasileiros de orientação vocacional e profissional: Período 1999-2009. Revista Brasileira de Orientação Profissional, v. 11, p. 107-120.

Leal, M. S.; Melo-Silva, L. L. ; Teixeira, M. O. (2015). Crenças para lidar com tarefas de carreira em estudantes do ensino médio. Avaliação Psicológica, 14(1), p. 125-132.

Marland, S. P. (1972). Career education: A handbook for implementation. Washington, DC: U. S. Department of Health Education and Welfare.

Melo-Silva, L. L. ; Munhoz, I. ; Leal, M. S. (2019). Orientação profissional na educação básica como política pública no Brasil. Revista Brasileira De Orientação Profissional, 20(1), p. 3-18.

Mohammad, J. (2016). Career maturity among university students in Jordan: The case for social studies. Australian Jorunal of Carrer Development, 25 (3), 110-1116. 
Munhoz, I.M. S., Melo-Silva, L. L., Audibert, A. (2016). Educação para a carreira pistas para intervenções na educação básica. In R. S. Levenfus (Org.), Orientação Vocacional e de Carreira em Contextos Clínicos e Educativos. Porto Alegre: Artmed.

Neiva, K. M. C. (1999). Escala de Maturidade para a Escolha Profissional (EMEP): Manual. São Paulo, SP: Vetor.

Noce, M. A. (2008). O BBT-Br e a maturidade para a escolha profissional: Evidências empíricas de validade (Tese de doutorado não publicada). Universidade de São Paulo, Ribeirão Preto, SP.

Patton, W., \& Lokan, J. (2001). Perspectives on Donald Super's Construct of Career Maturity. International Journal for Educational and Vocational Guidance, 1(1/2), 3148. Retirado de https://link.springer.com/article/10.1023\%2FA\%3A1016964629452. DOI:10.1023/A:1016964629452.

Savickas, M. L. (2004). Um modelo para avaliação de carreira. In L. M. Leitão (Org.), Avaliação psicológica em orientação escolar e profissional. Coimbra, Portugal: Quarteto.

Super, D. E. (1983). Assessment in career guidance: Toward truly developmental counseling. Personnel and Guidance Journal, 61(9), 555-562.

Super, D. E. (1985).Coming of age in middletown: careers in the making. American Psychologist, 40, 405-414.

Super, D. E. (1990). The life-span, life-space approach to career development. In D. Brown, L. Brooks \& Associates (Eds.), Career choice and development (2nd ed., pp. 197-261). San Francisco, CA: Jossey-Bass.

Talib, J. A., Salleh, A., Amat, S., Ghavifekr, S., Ariff, A. M. (2015). Effect of career education module on career development of community college students. International Journal for Educational and Vocational Guidance. 15(1), 37-55. https://doi.org/10.1007/s10775-014-9279-x 
Ulas, O. \& Yildirim, I. (2015). Predictors of Career Maturity among High School Students. Hacettepe Universitesi Egitim Fakultesi Dergisi-Hacettepe University Journal of Education, 30(2), 151-165.

Watts, A. G., \& Sultana, R. G. (2004). Career guidance policies in 37 countries: Contrasts and common themes. International Journal Educational Vocational Guidance, 4(2-3), $105-122$

Zahra, S. T. \& Malik, A. A. (2018). Relationship between Self-concept and Career Maturity in Pakistani High School Students. Bahria Journal of Professional Psychology, 17(1), 1-16. 